\title{
The efficacy of postoperative radiotherapy in localized primary soft tissue sarcoma treated with conservative surgery
}

\author{
Ru-Ping Zhao ${ }^{1 \dagger}$, Xiao-Li Yu ${ }^{2,3+}$, Zhen Zhang ${ }^{2,3}$, Li-Juan Jia ${ }^{4}$, Yan Feng ${ }^{2,3}$, Zhao-Zhi Yang ${ }^{2,3}$, Xing-Xing Chen ${ }^{2,3}$, \\ Jian Wang ${ }^{3,5}$, Sheng-Lin Ma ${ }^{6^{*}}$ and Xiao-Mao Guo ${ }^{2,3^{*}}$
}

\begin{abstract}
Background: To evaluate the efficacy of postoperative radiotherapy (RT) on local failure-free survival (LFFS), distant metastasis-free survival (DMFS) and overall survival (OS) in patients with localized primary soft tissue sarcoma (STS) and to identify prognostic factors.

Methods and materials: Between January 2000 and July 2010, 220 consecutive patients with localized primary STS, who received conservative surgery with or without postoperative RT, were enrolled in the study. Survival curves were constructed by the Kaplan-Meier method and log-rank test was used to assess statistical significance. Multivariate analysis was applied to identify the prognostic factors.

Results: After a median follow-up of 68 months (range, 5-127 months), the 5-year LFFS, DMFS and OS were 70.0, 78.2 and $71.2 \%$, respectively. Tumor size, histological subtypes, margin status and postoperative RT were independent predictors for OS. Postoperative RT was associated with a significant reduced local recurrence risk versus surgery alone (hazard ratio $[\mathrm{HR}]=0.408,95 \%$ confidence interval [CI] 0.235-0.707, $P=0.001$ ), with 5-year LFFS of 81.1 and $63.6 \%$, respectively (log-rank, $P=0.004$ ). The log-rank test showed that postoperative RT had a tendency of improving OS compared with surgery alone, with 5-year OS of 74.8 and $65.0 \%$, respectively $(P=0.089)$. Multivariate analysis demonstrated that postoperative RT significantly reduced mortality rate compared with surgery alone ( $H R=0.512$, $95 \% \mathrm{Cl} 0.296-0.886, p=0.017)$, especially in patients with liposarcoma $(p=0.034)$.
\end{abstract}

Conclusion: Postoperative radiotherapy reduce both local recurrence and STS mortality in patients with localized primary STS. The efficacy of RT on survival warrants further prospective study.

Keywords: Soft tissue sarcoma, Surgery, Radiotherapy, Local recurrence, Overall survival

\section{Background}

Soft tissue sarcomas (STS) are a heterogeneous group of uncommon neoplasms arising from mesenchymal tissues, accounting for less than $1 \%$ of all malignancies [1]. The conservative surgery is the most important primary treatment for patients with STS [2]. Close margin and even positive margin may be needed to avoid amputation or preserve critical neurovascular structures, which lead to

\footnotetext{
* Correspondence: mashenglin1211@sina.com; guoxm1800@163.com ${ }^{\dagger}$ Equal contributors

${ }^{6}$ Department of Radiation Oncology, Hangzhou First People's Hospital, 261 Huan Sha Road, Hangzhou, Zhejiang, China

2Department of Radiation Oncology, Cancer Hospital of Fudan University, 270 Dong An Road, Xuhui, Shanghai, China

Full list of author information is available at the end of the article
}

high risk of local failure in the surgical bed. The data from previous studies showed the local recurrence rate after conservative surgery alone was up to $30 \%[3,4]$. Randomized trials demonstrated that the addition of postoperative radiotherapy (RT) after conservative surgery could further reduce local recurrence when compared with surgery alone [5, 6]. Based on these studies, conservative surgery plus RT became the mainstay treatment modality in patients with STS.

Although randomized trials showed postoperative RT significantly improved local control, the local control benefit associated with RT did not translate into a survival advantage. Even after 17 years of follow-up, Beane JD et al. only demonstrated a trend toward improved 
survival in the RT group that failed to reach statistical significance. The authors acknowledged that their study was underpowered to demonstrate a survival advantage of $<20 \%$ [7]. This scenario has been replicated in several retrospective series [8-10]. It was initially believed that the improved local control by postoperative RT could only enhance local control, while had no efficacy on survival in patients with breast cancer. After accumulating the patients who treated with RT, The reports of the early Breast Cancer Trialists' Collaborative Group (EBCTCG) metaanalysis demonstrated that postoperative RT reduced the incidence of breast cancer recurrence and mortality [11]. It is likely that previous studies were underpowered to definitively demonstrate a survival advantage with the use of RT in patients with STS. Recently, several SEER analyses and retrospective studies that including a large number of STS reported that postoperative RT could also improve the survival of STS [12-15]. The question still remains if the improved local control with the use of RT can convert to survival benefit or not. The impacts of RT on patients with STS merit further investigation.

This paper summarized our experience with homogenous population of adult patients with STS, who were treated by the conservative modality, either surgery alone or surgery plus RT. The aim of the study is to evaluate the impacts of RT on local failure-free survival (LFFS) and distant metastasis-free survival (DMFS) and overall survival. This study also evaluates the relationship between various clinicopathologic factors and disease outcomes to identify the prognostic factors.

\section{Methods/materials}

\section{Patients}

We retrospectively reviewed 251 consecutive adult patients with localized primary STS during the period of January 2000 to July 2010. We excluded those patients with secondary sarcoma $(n=6)$, who received amputation or the treatment for palliative purpose $(n=8)$, surgically unresectable disease at presentation $(n=5)$, and those without follow-up data $(n=12)$. The remaining 220 patients were represented in this study. Histological diagnosis was confirmed in each case through review of the slides by a pathologist. The Institutional Review Board of Cancer Hospital of Fudan University approved review of data for this investigation.

The French Federation of Cancer Centers grading system was used for tumor grading, which was determined as low- (Grade I), intermediate- (Grade II) or high-grade (Grade III) [16]. A negative margin was defined as the absence of tumor at the inked margin. We reviewed the medical records, including operative and pathologic reports, and recorded the following information: age, gender, tumor anatomic location, histopathological subtype, size, grade, surgical margins status, lymph node status, treatment modality and toxicities.

\section{Treatment}

All patients were reviewed at an oncology multidisciplinary board and underwent appropriate structure- and functionpreserving surgical resection. In patients with the tumor adjacent to neurovascular bundles or critical structures, an attempt was made to obtain gross tumor free margin. Some patients received postoperative radiation and/or chemotherapy (generally doxorubicin and ifosfamide) based on prognostic factors predicting higher risk of local recurrence and distant metastasis. Radiotherapy was delivered through $8 \mathrm{MV}$ linear accelerator photon to the target volume by two opposed fields, in a schedule of $2 \mathrm{~Gy} /$ fraction, five fractions per week. An initial dose of 50Gy was delivered after surgical wound healing (within 8 weeks after surgery). Field borders were either the whole compartment, or proximal and distal margins of $5 \mathrm{~cm}$ in non-compartmental lesions and trunk. A cone-down field of the original tumor bed plus $3 \mathrm{~cm}$ margins received an additional boost (10-20Gy). Few patients did not receive full dose RT for protecting organs at risk adjacent radiation field.

\section{Follow-up}

The items of follow-up included physical examination, chest CT and ultrasonography of the primary site, with additional MRI when local recurrence was suspected. The patients were followed at 3-month interval for the first 2 years, 6-month interval for the following 3 years and yearly thereafter. The major endpoints of this study were local recurrence, distant metastasis and overall survival. Local recurrence was defined as the first pathological verified tumor of the same histological type, within or contiguous to the previously treated tumor bed at least 3 months after treatment. Distant metastasis was defined by clinical, pathological or radiologic evidence of systemic disease spread outside the primary tumor site.

\section{Toxicity}

Toxicities were evaluated by chart review using the Common Terminology Criteria for Adverse Events (CTCAE) version 3.0. Only wound complications were recorded in patients received surgery alone. The radiation-related toxicities were recorded in patients received RT. The highest grade of any observed toxicities reported for each patient at the time of follow-up. Only $\geq$ grade 2 toxicities were reported.

\section{Statistical analysis}

Descriptive data was compared using Pearson Chi-square test or Fisher's exact test, where appropriate. For survival analysis, survival curves were constructed using the 
Kaplan-Meier method. The effect of demographic, clinical, pathologic and treatment variables on survival were examined using the log-rank test to assess statistical difference. Multivariate analyses were performed using Cox logistic regression method and $P$-value of $<0.01$ was included into multivariate analysis to fully identify prognostic factors. A two-sided $P$-value of $<0.05$ determined statistical significance for all tests. All statistical analyses were performed using SPSS 17.0 software package.

\section{Results}

\section{Patient characteristics}

Patient, tumor, and treatment characteristics of study population were listed in Table 1 . The median age at diagnosis was 50 years (range, 18-86 years) and $60 \%$ of patients were male. Exactly half tumors (110 cases, $50.0 \%)$ were located in extremities, $52(23.6 \%)$ in trunk, $45(20.5 \%)$ in retroperitoneum and $13(5.9 \%)$ in head/ neck region. The most common histological subtype was liposarcoma (41.4\%). The median tumor size was $7 \mathrm{~cm}$ (range, 2-42 cm). RT was administered to 81 patients (36.8\%). The radiation dose ranged from $45 \mathrm{~Gy}$ to 70 Gy, with a median dose of 60 Gy.

The patients were divided into RT group and no RT group according to whether they received RT or not. The distribution of the patient characteristics was listed in Table 2. There were higher proportion of patients with tumors located in the extremities and head/neck, $\mathrm{MFH}$, grade III, positive margin and the usage of chemotherapy in RT group, while the no RT group had a higher rate of patients with tumors located in retroperitoneum, liposarcoma, grade I and negative margin. The distribution of other clinicopathological factors was not significantly different between two cohorts $(P>0.05)$.

\section{Endpoints and survival analysis Local failure-free survival}

With a median follow-up of 68 months (range, 5-127 months), a total of 72 patients experienced local recurrence. Fifty-three patients had only local recurrence as their initial site of failure, while in three patients, local recurrence and distant metastases were detected concurrently. Of 53 patients with local recurrence as initial site of treatment failure, the median time to local failure was 23 months (range, 3-126 months). The 5-year LFFS rate was $70.0 \%$. In the multivariate analysis for LFFS, tumor size, margin status and postoperative RT emerged as independent prognostic factors $(p<0.05)$. Postoperative RT significantly improved local control compared with surgery alone [hazard ratio $(\mathrm{HR})=0.408,95 \%$ confidence interval (CI) $0.235-0.707, P=0.001$, Table 3], with 5-year LFFS of 81.1 and $63.6 \%$, respectively (logrank, $P=0.004$, Fig. 1a). Larger tumor size $(\mathrm{HR}=1.815$, $95 \%$ CI 1.051-3.138 $p=0.033)$ and positive margin
Table 1 Patient, tumor and treatment characteristics $(n=240)$

\begin{tabular}{|c|c|c|}
\hline Characteristic & Number & Percent \\
\hline \multicolumn{3}{|l|}{ Age (years) } \\
\hline$\leq 50$ & 114 & 51.8 \\
\hline$>50$ & 106 & 48.2 \\
\hline \multicolumn{3}{|l|}{ Gender } \\
\hline Male & 132 & 60.0 \\
\hline Female & 88 & 40.0 \\
\hline \multicolumn{3}{|l|}{ Location } \\
\hline Extremity & 110 & 50.0 \\
\hline Trunk & 52 & 23.6 \\
\hline Head/neck & 45 & 20.5 \\
\hline Retroperitoneum & 13 & 5.9 \\
\hline \multicolumn{3}{|l|}{ Histology } \\
\hline Rhabdomyosarcoma & 27 & 12.3 \\
\hline Fibrosarcoma & 23 & 10.5 \\
\hline MFH & 54 & 24.5 \\
\hline Liposarcoma & 90 & 40.9 \\
\hline Others & 26 & 11.8 \\
\hline \multicolumn{3}{|l|}{ Tumor size (cm) } \\
\hline$\leq 5$ & 80 & 36.4 \\
\hline$>5$ & 140 & 63.6 \\
\hline \multicolumn{3}{|l|}{ Grade } \\
\hline 1 & 31 & 14.1 \\
\hline$\|$ & 58 & 26.4 \\
\hline III & 131 & 59.5 \\
\hline \multicolumn{3}{|l|}{ Margin status } \\
\hline Negative & 195 & 88.6 \\
\hline Positive & 25 & 11.4 \\
\hline \multicolumn{3}{|l|}{ Lymph node status } \\
\hline Negative & 210 & 95.5 \\
\hline Positive & 10 & 4.5 \\
\hline \multicolumn{3}{|l|}{ Chemotherapy } \\
\hline No & 179 & 81.4 \\
\hline Yes & 41 & 18.6 \\
\hline \multicolumn{3}{|l|}{ Radiotherapy } \\
\hline No & 139 & 63.2 \\
\hline Yes & 81 & 36.8 \\
\hline
\end{tabular}

$(\mathrm{HR}=2.595,95 \%$ CI $1.432-4.702 p=0.002)$ correlated with worse outcome. The other clinicopathological factors were not significant in model.

\section{Distant metastasis-free survival}

Forty-eight patients developed distant metastases in this study, including 30 patients with distant metastases only and 18 patients with distant metastases and local recurrence as well. Of the 30 patients with distant metastases 
Table 2 Distribution of patient, tumor and treatment characteristics by receiving RT or not

\begin{tabular}{|c|c|c|c|c|c|}
\hline \multirow[b]{2}{*}{ Characteristic } & \multicolumn{2}{|l|}{ RT } & \multicolumn{2}{|c|}{ No RT } & \multirow[b]{2}{*}{$P$ Value* } \\
\hline & No. & $\%$ & No. & $\%$ & \\
\hline Age (years) & & & & & 0.786 \\
\hline$\leq 50$ & 41 & 50.6 & 73 & 52.5 & \\
\hline$>50$ & 40 & 49.4 & 66 & 45.5 & \\
\hline Gender & & & & & 0.967 \\
\hline Male & 49 & 60.5 & 83 & 59.7 & \\
\hline Female & 32 & 39.5 & 56 & 40.3 & \\
\hline \multicolumn{6}{|l|}{ Location } \\
\hline Extremity & 53 & 65.4 & 57 & 41.0 & 0.001 \\
\hline Trunk & 15 & 18.5 & 37 & 26.6 & 0.191 \\
\hline Head/neck & 11 & 13.6 & 2 & 1.4 & $<0.001$ \\
\hline Retroperitoneum & 2 & 2.5 & 43 & 30.9 & $<0.001$ \\
\hline \multicolumn{6}{|l|}{ Histology } \\
\hline Rhabdomyosarcoma & 12 & 14.8 & 15 & 10.8 & 0.400 \\
\hline Fibrosarcoma & 8 & 9.9 & 15 & 10.8 & 0.831 \\
\hline MFH & 28 & 34.6 & 26 & 18.7 & 0.010 \\
\hline Liposarcoma & 22 & 27.2 & 68 & 48.9 & 0.002 \\
\hline Others & 11 & 13.6 & 15 & 10.8 & 0.525 \\
\hline Tumor size (cm) & & & & & 0.773 \\
\hline$\leq 5$ & 30 & 37.0 & 50 & 36.0 & \\
\hline$>5$ & 51 & 63.0 & 89 & 64.0 & \\
\hline \multicolumn{6}{|l|}{ Grade } \\
\hline । & 4 & 4.9 & 27 & 19.4 & 0.002 \\
\hline$\|$ & 21 & 25.9 & 37 & 26.6 & 1.000 \\
\hline III & 56 & 69.1 & 75 & 54.0 & 0.033 \\
\hline Margin status & & & & & 0.029 \\
\hline Negative & 66 & 81.5 & 128 & 92.1 & \\
\hline Positive & 15 & 18.5 & 11 & 7.9 & \\
\hline Lymph node status & & & & & 0.177 \\
\hline Negative & 75 & 92.6 & 135 & 97.1 & \\
\hline Positive & 6 & 7.4 & 4 & 2.9 & \\
\hline Chemotherapy & & & & & 0.019 \\
\hline No & 59 & 72.5 & 120 & 86.3 & \\
\hline Yes & 22 & 27.2 & 19 & 13.7 & \\
\hline
\end{tabular}

Abbreviation: $R T$ radiotherapy

*Determined by chi-square test

as initial site of treatment failure, the median time to distant metastasis was 15 months (range, 3-114 months). The 5-year DMFS rate was $78.2 \%$. The multivariate analysis showed that the significant predictor for distant metastasis was only histological subtype. Rhabdomyosarcoma correlated with worst outcome, while fibrosarcoma and liposarcoma predicted for better DMFS, with HR of 0.148
(95\% CI $0.033-0.661, p=0.012)$ and 0.126 (95 \% CI $0.056-0.303, p<0.001$, Table 3$)$, respectively. Although univariate analysis showed grade, lymph node status and chemotherapy were significant predictors for distant metastasis, these factors lost significant in the following Cox logistic regression analysis (Additional file 1: Table S1).

\section{Overall survival}

A total of 65 deaths occurred during follow-up, including 60 patients who died of sarcoma-related causes. Other causes of death included stroke (one patient), respiratory failure (one), heart failure (one) and second cancers (two). Of the 60 patients who died of sarcoma-related causes, 19 patients developed local recurrence only, 18 patients developed distant metastases only and 23 patients developed both local recurrence and distant metastases. The 5-year OS rate was $71.2 \%$. The univariate analysis showed RT had a tendency of improving OS, with 5-year OS of 74.8 and $65.0 \%$ in the RT group and no RT group, respectively $(P=0.089$, Fig. 1c). The multivariate analysis demonstrated that RT was associated with improved OS $(\mathrm{HR}=0.512,95 \%$ CI 0.296-0.886, $p=0.017$, Table 3). In the multivariate analysis, tumor size $>5 \mathrm{~cm}$ was also an independent significant adverse prognostic factor compared to tumor size $\leq 5 \mathrm{~cm}$, with a $\mathrm{HR}$ of 2.638 (95 \% CI 1.392-4.998, $p=0.003$ ). Additional adverse prognostic factor was positive margin status $(\mathrm{HR}=3.942$, 95 \% CI 2.233-6.959, $p<0.001)$. Rhabdomyosarcoma was the worst histological subtype. Comparison with rhabdomyosarcoma, patients with liposarcoma $(\mathrm{HR}=0.186,95 \%$ CI $0.088-0.392, p<0.001)$ and fibrosarcoma $(\mathrm{HR}=0.142,95 \%$ CI $0.039-0.510, p=0.003)$ were associated with significant better survival.

\section{The subgroup analysis on radiation effect}

Local recurrence was developed in 21 of 81 patients (25.9 \%) who received RT and 51 of 139 patients (36.7 \%) who did not received RT, respectively. In RT group, 13 patients developed local recurrence alone, 15 patients developed distant metastases alone, and eight patients developed both local and distant failure. In no RT group, 40 patients developed local failure alone, 14 patients developed distant metastases alone and 11 patients experienced both local and distant failure. Postoperative RT significantly improved local control compared with no postoperative RT ( $\mathrm{HR}=$ 0.408, $95 \%$ CI $0.235-0.707, P=0.001$, Table 3 ), with a 5 -year actuarial LFFS of 81.1 and $63.6 \%$, respectively (log-rank, $P=0.004$, Fig. 1a). The median time to local recurrence in RT group and no RT group were 29 months (range, 4-126 months) and 15 months (range, 3-120 months), respectively. The log-rank test showed that RT postponed the time to local recurrence in patients who experienced local recurrence $(P=0.022)$. RT had no impact 
Table 3 Multivariate analysis for LFFS, DMFS and OS

\begin{tabular}{|c|c|c|c|c|c|c|}
\hline \multirow[b]{2}{*}{ Characteristic } & \multicolumn{2}{|l|}{ LFFS } & \multicolumn{2}{|l|}{ DMFS } & \multicolumn{2}{|l|}{ OS } \\
\hline & HR $(95 \%$ Cl) & $P^{*}$ & HR (95\% Cl) & $p$ & HR $(95 \%$ Cl) & \\
\hline Age (>50 vs. $\leq 50)$ & & 0.980 & & 0.774 & & 0.210 \\
\hline Gender & & 0.602 & & 0.780 & & 0.794 \\
\hline Location & & 0.560 & & 0.453 & & 0.506 \\
\hline Histology & & 0.621 & & $<0.001$ & & $<0.001$ \\
\hline Rhabdomyosarcoma & & & 1.00 & & 1.00 & \\
\hline Fibrosarcoma & & & $0.128(0.029-0.569)$ & 0.012 & $0.142(0.039-0.510)$ & 0.003 \\
\hline MFH & & & $0.574(0.281-1.174)$ & 0.088 & $0.516(0.254-1.047)$ & 0.067 \\
\hline Liposarcoma & & & $0.161(0.069-0.378)$ & $<0.001$ & $0.186(0.088-0.392)$ & $<0.001$ \\
\hline Others & & & $0.407(0.154-1.074)$ & 0.074 & $0.426(0.169-1.073)$ & 0.070 \\
\hline Tumor size (cm) & & 0.033 & & 0.066 & & 0.003 \\
\hline$\leq 5$ & 1.00 & & & & 1.00 & \\
\hline$>5$ & 1.815 (1051-3.138) & & & & 2.638 (1.392-4.998) & \\
\hline Grade & & 0.147 & & 0.259 & & 0.400 \\
\hline Margin status & & 0.002 & & 0.143 & & $<0.001$ \\
\hline Negative & 1.00 & & 1.00 & & 1.00 & \\
\hline Positive & $2.595(1.432-4.702)$ & & $2.187(1.055-4.536)$ & & $3.942(2.233-6.959)$ & \\
\hline Lymph node status & & 0.909 & & 0.272 & & 0.582 \\
\hline Chemotherapy & & 0.687 & & 0.894 & & 0.719 \\
\hline Radiotherapy & & 0.001 & & 0.769 & & 0.017 \\
\hline No & 1.00 & & & & 1.00 & \\
\hline Yes & $0.408(0.235-0.707)$ & & & & $0.512(0.296-0.886)$ & \\
\hline
\end{tabular}

Abbreviations: HR hazard ratio, Cl confidence interval, OS overall survival, LFFS local failure-free survival, DMFS distant metastasis-free survival

*Determined by Cox logistic regression method

on the risk of development distant metastasis, with 5-year DMFS of 73.1 and $80.8 \%$ in the RT group and no RT group, respectively $(P=0.307$, Fig. $1 b)$.

When we examined the impact of RT on OS, we noted a tendency of improving OS, with 5-year OS of 74.8 and $65.0 \%$ in the RT group and no RT group, respectively $(P=0.089$ Fig. $1 \mathrm{c})$. The multivariate analysis demonstrated that RT was associated with improved OS (HR $=0.512,95 \%$ CI, 0.296-0.886, $p=0.017$, Table 3). Only two patients with retroperitoneal STS received RT in our study, which might bias the final survival results. We excluded these patients with retroperitoneal STS and reanalyzed the efficacy of RT on OS. Multivariate analysis confirmed that RT improved the OS ( $\mathrm{HR}=0.450,95 \% \mathrm{CI}$, $0.243-0.833, P=0.011$ ). We also performed univariate analyses to identify specific subgroups of patients that derived benefit from RT (Additional file 1: Table S2). The result showed that RT significantly improved the 5-year OS in the subset of patients with liposarcoma (90.9 \% vs. $76.5 \%, p=0.041$, Fig. 1d).

\section{Toxicity}

Sixteen of 139 patients (11.5\%) in the no RT group compared to 13 of 81 patients $(16.0 \%)$ in the RT group had $\geq$ grade 2 wound complications. In the patients received $\mathrm{RT}, 31$ (38.3\%) patients suffered from $\geq$ grade 2 radiation dermatitis. Late $\geq$ grade 2 radiation-related toxicities were recorded including peripheral nerve damage, edema, joint stiffness and bone fracture. One (1.2\%) patient developed radiation-related peripheral nerve damage, 10 (12.3\%) patients had edema, 8 (9.9\%) patients suffered from joint stiffness and bone fractures were not seen.

\section{Discussions}

Our result showed that postoperative RT significantly reduced the risk of local recurrence, while had no impact on development of distant metastasis. When we examined the impact of postoperative RT on OS, we noted RT had a tendency of improving OS. Multivariate analysis demonstrated that RT was a significant prognostic factor for OS.

Randomized trials had demonstrated that postoperative radiotherapy could improve local control in patients with STS when compared to surgery alone, especially in patients with high grade tumor. Yang et al. showed that postoperative RT significantly decreased the 10-year local recurrence rate among patients with high-grade 

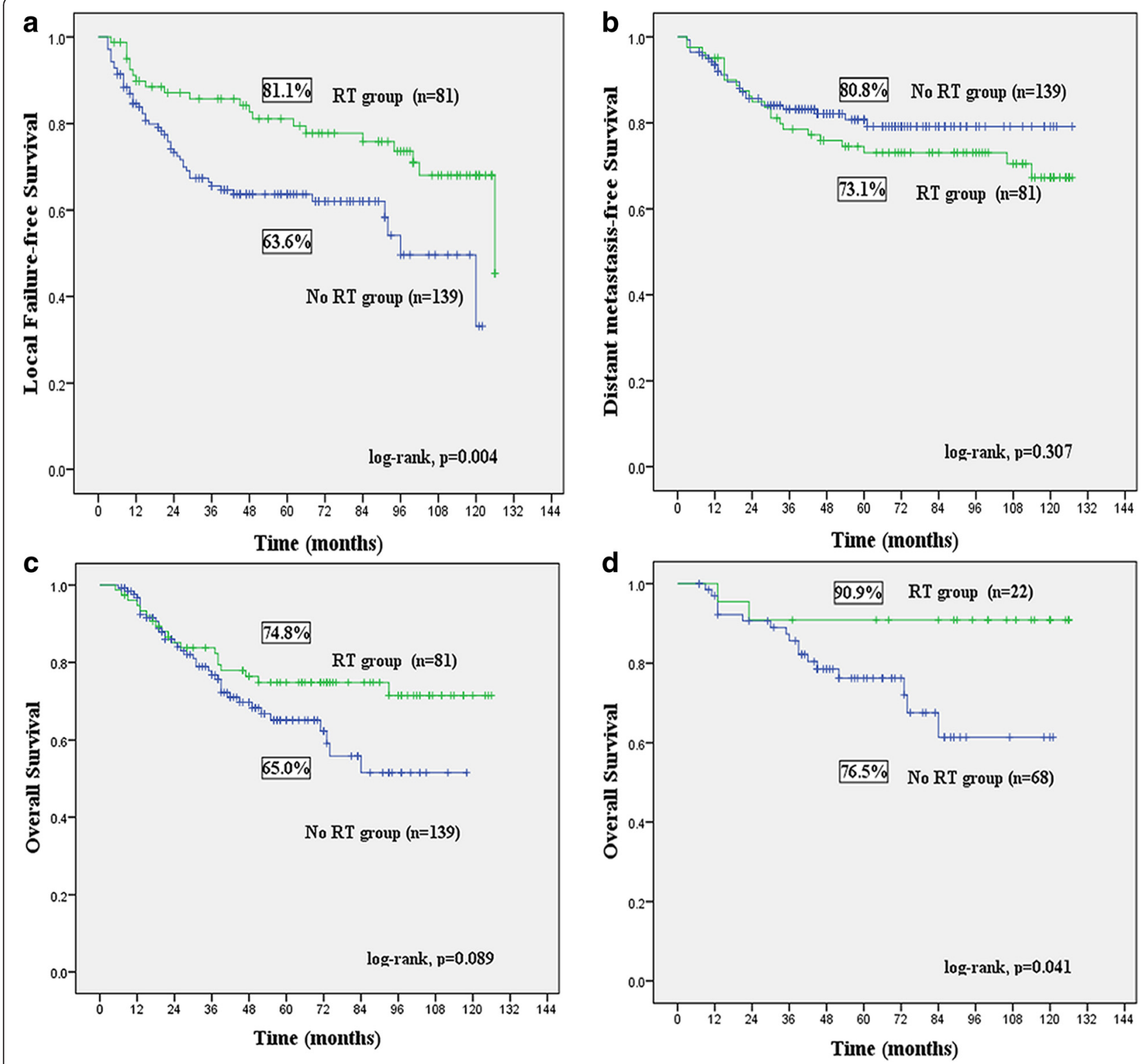

Fig. 1 Kaplan-Meier estimates comparing patients receiving RT (RT group) and without receiving RT (No RT group) for a local failure-free survival; b diatant metastasis-free survival; c overall survival; d overall survival in liposarcoma

lesions (no local recurrence in the RT group Vs $22 \%$ in no RT group, $p=0.0028$ ) [6]. In another study, Adjuvant brachytherapy improves local control after complete resection of soft tissue sarcomas in patients with high-grade tumors, with the 5 -year local control rate were 89 and $66 \%$ in the RT and no RT group $(p=0.0025)$, respectively [5]. The data from other retrospective studies also demonstrated that RT could improve local control in patients with STS [8-10, 17, 18]. In our study, the effect of RT on local control was in line with previous reports. Although the impact of radiation on local control for extremity sarcoma has been well studied, the effects of local control on survival are less well understood.
Multiple variety studies have examined the question of survival benefit for postoperative RT in STS. However, few of those studies had sufficiently power to detect a survival advantage. Recently, several studies observed that RT improved the survival of STS. In a study including 6960 patients with STS, Koshy et al. reported that the 3-year overall survival rate was $73 \%$ in the radiation group compared with $63 \%$ in the no radiation group $(p<0.001)$. RT was associated with a significantly improved OS (HR $=0.78,95 \% \mathrm{CI}, 0.69-0.89)$ [12]. Schreiber et al. analyzed a cohort of 983 patients with high-grade sarcoma. The authors found a survival benefit at 3 years for patients with high-grade tumors $>5 \mathrm{~cm}$ 
treated with radiotherapy (73.4 vs. $55.6 \%, P<0.001)$ [19]. Kachare et al. found that RT was associated with a $5 \% 5$ year survival advantage in a study including 2606 patients with high-grade sarcoma of the extremity. The authors concluded radiotherapy, regardless of the timing, was associated with improved survival in high-risk sarcoma [15]. In another study, Gutierrez et al. observed a statistically significant increase in median overall survival of 3 months in patients undergoing RT $(p<0.001)$. Administration of RT for high-grade lesions increased median survival to 25 months compared to only 16 months when RT was not used $(p<0.001)$ [20]. Alkis et al. found postoperative RT was associated with significant improved OS in a retrospective study including 294 patients with STS, with 5-year OS were $62.2 \%$ and $32.1 \%(P<0.0001)$ in patients who received postoperative $\mathrm{RT}$ and surgery alone, respectively [13]. Another retrospective analysis of 202 patients with high grade STS of the extremity, brachytherapy following limb-sparing surgery resulted in favorable 5-year local control and OS rates [14]. All the previous studies indicated RT could improve the survival of patients with STS, which was in line with our results.

The scenario of RT improves survival can be interpreted as the improvement of local control by RT translates into survival benefit. In fact, the relationship between local control and prognosis of STS has long been debated. There were many published studies showed that patients with local recurrence had worse prognosis. The rationale may be illustrated as follow. Firstly, RT may prevent further tumor seeding by reducing local recurrence. Lewis et al. analyzed the correlation of local recurrence with subsequent metastases in 911 patients with extremity STS. The metastasis after local recurrence significantly increased in patients with high-grade and deep tumors. They concluded that there was a strong association of local recurrence with the development of subsequent metastasis and tumor mortality [21]. Secondly, although the majority patients with STS died of distant metastasis, local recurrence can also directly influence survival. Gronchi et al. observed nearly $20 \%$ of patients with STS receiving R1 resection died of loco-regional recurrences without developing distant metastases [22]. Lewis et al. reported 84 of 112 (75\%) patients with primary retroperitoneal STS died in the absence of distant metastasis. The local recurrence rather than distant metastasis was the primary cause of death [23]. A study from M. D. Anderson Cancer Center (MDACC) reported that a significant fraction of patients who died of STS (46 of 372 patients, $12 \%$ ) had only local recurrence. The authors believed that local recurrence was likely to influence disease specific survival as well [24]. In our study, of 60 patients who died of cancer-related causes, 19 patients (32\%) died of local recurrence without developing distant metastasis. The improvement of local control by using of RT has potential take survival benefit in those patients.
Sequencing of radiotherapy may also affect outcomes in patients with STS. The only phase III randomized study comparing preoperative to postoperative RT was conducted by O'Sullivan et al. [25]. One hundred and ninety patients were stratified by tumor size and randomized to preoperative (94 patients) or postoperative RT (96 patients). Patients who had preoperative RT had more wound complications than those receiving postoperative RT after treatment (35\% vs. $17 \%, P=0.01$ ). However, at a median follow-up of 6.9 years, patients treated with preoperative RT had a lower frequent of subcutaneous fibrosis ( $31.5 \%$ vs. $48.2 \%, P=0.07)$, joint stiffness ( $17.8 \%$ vs. $23.2 \%, P=0.51)$, and edema ( $15.1 \%$ vs. $23.2 \%$, $P=0.26$ ). There was no difference in terms of survival or local, regional, and distant failure rates between the two delivery methods [26]. Since acute wound complications can be managed and are generally temporary whereas late toxicities are longer lasting, preoperative RT is increasingly favored.

Histology, size, margin status and RT were identified as significant prognostic factors for OS in our study, which was generally in line with other reports. Stojadinovic et al. identified that tumor size, grade, and resection margin were significantly associated with sarcoma specific survival in 2123 patients with completely resected localized primary STS [27]. Pisters et al. reported that tumor size, grade, the histology, margin status and location were the prognostic factors for disease specific survival [28]. Zagars et al. studied the prognostic factors for disease specific survival in patients with localized STS treated with conservative surgery and RT. They found that the independent factors that affected disease specific survival were tumor grade, tumor size, tumor site, histopathology, patient age, and resection margins [24]. MarettyNielsen et al. established that age, size, grade, margin, and radiotherapy were important prognostic factors for both local recurrence and disease specific mortality in a cohort study of 922 consecutive patients with STS [29].

\section{Conclusion}

STS is a disease largely limited to retrospective reports given the paucity incidence. It is hard to study this disease systematically and therefore little information was provided to assess the long-term benefits of RT. The current study presented the outcomes of a single institution analysis of 220 patients with localized primary STS treated by conservative strategy. Our study showed that RT reduced local recurrence and had potential effect to improve OS. With the limitations of a retrospective database study, the results of our study suggested that aggressive local therapy with RT followed by definitive surgery may provide patients the opportunity for improved long-term survival. Prospective studies examining the efficacy of RT on survival are necessary. 


\section{Additional file}

Additional file 1: Table S1. Comparison of patient, tumor and treatment factors for 5-year LFFS, DMFS and OS. Table S2. The subgroup analysis of the impact of RT on OS. (DOCX $27 \mathrm{~kb}$ )

\section{Abbreviations}

Cl: confidence interval; DMFS: distant metastasis-free survival; HR: hazard ratio; IMRT: intensity-modulated radiation therapy; LFFS: local failure-free survival; OS: overall survival; RT: radiotherapy; STS: soft tissue sarcoma.

\section{Competing interests}

The authors declare that they have no competing interests.

\section{Authors' contributions}

R-PZ and X-LY carried out data collection, participated in analysis, helped draft manuscript. ZZ, YF and carried out analysis and helped draft manuscript. L-JJ involved in conceiving the study and participated in analysis. JW reviewed the slides. Z-ZY and X-XC assisted with data collection. SLM and X-MG designed the protocol, conceived he study and helped to draft the manuscript. All authors read and approved the final manuscript.

\section{Author details}

'Department of Radiation Oncology, Hangzhou Cancer Hospital, 34 Yan Guan Lane, Hangzhou, Zhejiang, China. ${ }^{2}$ Department of Radiation Oncology, Cancer Hospital of Fudan University, 270 Dong An Road, Xuhui, Shanghai, China. ${ }^{3}$ Department of Oncology, Shanghai Medical College of Fudan University, 270 Dong An Road, Xuhui, Shanghai, China. ${ }^{4}$ Department of medical Oncology, Shandong Binzhou Central Hospital, 108 Southern Huancheng Road, Binzhou, Shandong, China. ${ }^{5}$ Department of Pathology, Cancer Hospital of Fudan University, 270 Dong An Road, Xuhui, Shanghai, China. ${ }^{6}$ Department of Radiation Oncology, Hangzhou First People's Hospital, 261 Huan Sha Road, Hangzhou, Zhejiang, China.

Received: 13 October 2015 Accepted: 18 February 2016

Published online: 25 February 2016

\section{References}

1. Jemal A, Siegel R, Xu J, Ward E. Cancer statistics, 2010. CA Cancer J Clin. 2010:60:277-300

2. DeLaney TF, Kepka L, Goldberg SI, Hornicek FJ, Gebhardt MC, Yoon SS, et al. Radiation therapy for control of soft-tissue sarcomas resected with positive margins. Int J Radiat Oncol Biol Phys. 2007;67:1460-69.

3. Barr LC, Stotter AT, A'Hern RP. Influence of local recurrence on survival: a controversy reviewed from the perspective of soft tissue sarcoma. Brit J Surg. 1991;78:648-50.

4. Cantin J, McNeer GP, Chu FC, Booher RJ. The problem of local recurrence after treatment of soft tissue sarcoma. Ann Surg. 1968;168:47.

5. Pisters PW, Harrison LB, Leung DH, Woodruff JM, Casper ES, Brennan MF. Long-term results of a prospective randomized trial of adjuvant brachytherapy in soft tissue sarcoma. J Clin Oncol. 1996;14:859-68.

6. Yang JC, Chang AE, Baker AR, Sindelar WF, Danforth DN, Topalian SL, et al. Randomized prospective study of the benefit of adjuvant radiation therapy in the treatment of soft tissue sarcomas of the extremity. J Clin Oncol. 1998; 16:197-203.

7. Beane JD, Yang JC, White D, Steinberg SM, Rosenberg SA, Rudloff U. Efficacy of adjuvant radiation therapy in the treatment of soft tissue sarcoma of the extremity: 20-year follow-up of a randomized prospective trial. Ann Surg Oncol. 2014:21:2484-89.

8. Alektiar KM, Velasco J, Zelefsky MJ, Woodruff JM, Lewis JJ, Brennan MF. Adjuvant radiotherapy for margin-positive high-grade soft tissue sarcoma of the extremity. Int J Radiat Oncol Biol Phys. 2000:48:1051-58.

9. Jebsen NL, Trovik CS, Bauer HC, Rydholm A, Monge OR, Hall KS, et al. Radiotherapy to improve local control regardless of surgical margin and malignancy grade in extremity and trunk wall soft tissue sarcoma: a Scandinavian sarcoma group study. Int J Radiat Oncol Biol Phys. 2008;71: 1196-203.

10. Krasin MJ, Davidoff AM, Xiong X, Wu S, Hua C, Navid F, et al. Preliminary results from a prospective study using limited margin radiotherapy in pediatric and young adult patients with high-grade nonrhabdomyosarcoma soft-tissue sarcoma. Int J Radiat Oncol Biol Phys. 2010;76:874-78.

11. McGale P, Taylor C, Correa C, Cutter D, Duane F, Ewertz M, et al. Effect of radiotherapy after mastectomy and axillary surgery on 10-year recurrence and 20-year breast cancer mortality: meta-analysis of individual patient data for 8135 women in 22 randomised trials. Lancet. 2014;383:2127-35.

12. Koshy M, Rich SE, Mohiuddin MM. Improved survival with radiation therapy in high-grade soft tissue sarcomas of the extremities: a SEER analysis. Int J Radiat Oncol Biol Phys. 2010;77:203-9.

13. Alkis N, Muallaoğlu S, Koçer M, Arslan ÜY, Durnalı AG, Tokluoğlu S, et al. Primary adult soft tissue sarcomas: analysis of 294 patients. Med Oncol. 2011;28:391-6

14. Alektiar KM, Leung D, Zelefsky MJ, Healey JH, Brennan MF. Adjuvant brachytherapy for primary high-grade soft tissue sarcoma of the extremity. Ann Surg Oncol. 2002:9:48-56.

15. Kachare SD, Brinkley J, Vohra NA, Zervos EE, Wong JH, Fitzgerald TL. Radiotherapy associated with improved survival for high-grade sarcoma of the extremity. J Surg Oncol. 2015;112:338-43.

16. Guillou L, Coindre J, Bonichon F, Nguyen BB, Terrier P, Collin F, et al. Comparative study of the National Cancer Institute and French Federation of Cancer Centers Sarcoma Group grading systems in a population of 410 adult patients with soft tissue sarcoma. J Clin Oncol. 1997:15:350-62.

17. Coindre J, Terrier P, Bui NB, Bonichon F, Collin F, Le Doussal V, et al. Prognostic factors in adult patients with locally controlled soft tissue sarcoma. A study of 546 patients from the French Federation of Cancer Centers Sarcoma Group. J Clin Oncol. 1996:14:869-77.

18. Wilson AN, Davis A, Bell RS, O'Sullivan B, Catton C, Madadi F, et al. Local control of soft tissue sarcoma of the extremity: the experience of a multidisciplinary sarcoma group with definitive surgery and radiotherapy. Eur J Cancer. 1994;30:746-51.

19. Schreiber D, Rineer J, Katsoulakis E, Sroufe RL, Lange CS, Nwokedi E, et al. Impact of postoperative radiation on survival for high-grade soft tissue sarcoma of the extremities after limb sparing radical resection. Am J Clin Oncol. 2012;35:13-7

20. Gutierrez JC, Perez EA, Franceschi D, Moffat Jr FL, Livingstone AS, Koniaris LG. Outcomes for soft-tissue sarcoma in 8249 cases from a large state cancer registry. J Surg Res. 2007:141:105-14.

21. Lewis JJ, Leung D, Heslin M, Woodruff JM, Brennan MF. Association of local recurrence with subsequent survival in extremity soft tissue sarcoma. J Clin Oncol. 1997;15:646-52.

22. Gronchi A, Vullo SL, Colombo C, Collini P, Stacchiotti S, Mariani L, et al. Extremity soft tissue sarcoma in a series of patients treated at a single institution: local control directly impacts survival. Ann Surg. 2010;251:506-11.

23. Lewis JJ, Leung D, Woodruff JM, Brennan MF. Retroperitoneal soft-tissue sarcoma: analysis of 500 patients treated and followed at a single institution. Ann Surg. 1998:228:355-65.

24. Zagars GK, Ballo MT, Pisters PW, Pollock RE, Patel SR, Benjamin RS, et al. Prognostic factors for patients with localized soft-tissue sarcoma treated with conservation surgery and radiation therapy. Cancer. 2003;97:2530-43.

25. O'Sullivan B, Davis AM, Turcotte R, Bell R, Catton C, Chabot P, et al. Preoperative versus postoperative radiotherapy in soft-tissue sarcoma of the limbs: a randomised trial. Lancet. 2002;359:2235-41.

26. Davis AM, O'Sullivan B, Turcotte $R$, Bell $R$, Catton C, Chabot $P$, et al. Late radiation morbidity following randomization to preoperative versus postoperative radiotherapy in extremity soft tissue sarcoma. Radiother Oncol. 2005;75:48-53.

27. Stojadinovic A, Leung DH, Allen P, Lewis JJ, Jaques DP, Brennan MF. Primary adult soft tissue sarcoma: time-dependent influence of prognostic variables. J Clin Oncol. 2002:20:4344-52.

28. Pisters PW, Leung DH, Woodruff J, Shi W, Brennan MF. Analysis of prognostic factors in 1,041 patients with localized soft tissue sarcomas of the extremities. J Clin Oncol. 1996;14:1679-89.

29. Maretty-Nielsen $\mathrm{K}$, Aggerholm-Pedersen N, Safwat A, Jørgensen PH, Hansen BH, Baerentzen $\mathrm{S}$, et al. Prognostic factors for local recurrence and mortality in adult soft tissue sarcoma of the extremities and trunk wall: a cohort study of 922 consecutive patients. Acta Orthop. 2014;85:323-32. 